\title{
LARGE INCIDENTAL GASTROINTESTINAL STROMAL TUMOR OF THE STOMACH
}

\author{
Irina Liepina*, Sergejs Klimcuks*, Jelena Farbere*, Ilona Kapeika** \\ * Riga lst Hospital, Departmen of Surgery, Riga, Latvia \\ ** Riga lst Hospital, Departmen of Radiology, Riga, Latvia
}

\section{Summary}

Gastrointestinal stromal tumors (GISTs) are the most common mesenchymal neoplasms of the gastrointestinal (GI) tract (3). A 68-year-old male patient was referred to our hospital with complaints about feeling pressure in the thorax and heavy sweating for over 2 years. Abdominal CT showed a pathological formation approximately $21 \mathrm{~cm} \times 15 \mathrm{~cm} \times 15 \mathrm{~cm}$ in size. The stomach was compressed and dislocated by the tumor. The total gastrectomy Roux modification was performed. The tumor grows from the back wall of the stomach; the tumor is not grew into the mucosa. We made a final diagnosis of an extra luminal GIST of the stomach - pT4NOMOG1R0 stage II. There was no evidence of disease recurrence six months after surgery.

Keywords: gastrointestinal stromal tumor, stomach, surgery

\section{AIM OF THE DEMONSTRATION}

Gastrointestinal stromal tumors (GISTs) are the most common mesenchymal neoplasms of the gastrointestinal (GI) tract (4). GISTs are more often located in the stomach $(56 \%)$ followed by small bowel $(32 \%)$, colorectum $(6 \%)$, and esophagus $(<1 \%)$. Sporadically, it may affect the omentum, mesentery, and peritoneum (7). GISTs are usually found in adults between ages 40 and 70; rarely, children and young adults develop these tumors (3). We present case report of large GIST with non-specific symptoms. GISTs tend to displace adjacent structures rather than invade them, and because of their submucosal origin, they can reach large sizes before becoming symptomatic. As a result, surgical treatment become more difficult.

\section{CASE REPORT}

A 68-year-old male patient was referred to our hospital with complaints about feeling pressure in the thorax and heavy sweating for over 2 years. He lost 5 kilograms of weight in six months. During this time the patient was checked for the presence of cardiovascular disease. The abdominal cavity was not examined. There is no oncology history in the patient's family. The holecistectomy was performed in 2008. Endoscopic examination of the stomach revealed no pathology in 2016.

Physical examination showed tenderness in the mid and upper right abdomen. A mass was palpated in the upper abdomen under the sternum. The findings were otherwise unremarkable. Laboratory findings including blood chemistries, routine blood tests, coagulation profile, and tumor markers were normal. Abdominal ultrasonography revealed a smoothly contoured heterogeneous with good vascularisation mass, $20 \mathrm{~cm} \times 14 \mathrm{~cm}$ in size. Abdominal CT showed a pathological formation approximately $21 \mathrm{~cm} \times 15 \mathrm{~cm} \times 15 \mathrm{~cm}$ in size (Fig.1.). The tumor gets a blood supply from a. gastrica sinistra and a. lienalis. The stomach was compressed and dislocated by the tumor (Fig.2.). The pancreatic gland was flipped down by the tumor. The border between the back wall of the stomach and the tumor was indistinct (Fig.3.). Gastric endoscopy revealed external compression.

The patient was prepared for surgical treatment. The total gastrectomy Roux modification was performed. The tumor grows from the back wall of the stomach; the tumor is not grew into the mucosa. It measured $22 * 15 * 21 \mathrm{~cm}$ in size and weighed $4.1 \mathrm{~kg}$ (Fig.4). Histopathological examination showed evidence of growth of spindle-shaped cells and a low mitotic index - <5/50 HPF. An immunohistochemical examination showed positive immunoreactions for CD34, CD117, but negative for cytokeratin. There were four lymph nodes in epiploon without metastases. Risk group of recurrence $3 \mathrm{~b}-12 \%$ (Miettinen and Lasota risk table for GIST). We made a final diagnosis of an extra luminal GIST of the stomach - pT4NOMOGIR0 stage II. The patient received parenteral nutrition because blood protein level was reduced after surgery. The post-operative course was without complications, and the patient was discharged on $14^{\text {th }}$ postoperative day. Chemotherapy was not needed.

\section{DISCUSSION}

A high proportion of GISTs are asymptomatic. They are frequently discovered incidentally during an endoscopic study or on radiological images obtained for another purpose. Incidental findings can cause a significant diagnostic delay. Currently, a significant number of patients present with metastases at the time of diagnosis (up to $50 \%$ in some cases)(1). However diagnostic delay was approximately two years and the tumor was very large, no evidence of metastases in our case. CT is the imaging modality of choice for diagnosis and staging of GISTs at initial presentation and for 
monitoring the disease during and after treatment (9). Surgery is the only potentially curative treatment for suspected resectable GIST. The primary objective of this process is to ensure that clear resection margins are obtained in a complete resection of the tumor, and it can be extirpated without tumor pseudocapsule rupture (5). We performed total gastrectomy Roux modification with clear resection margins. The operation lasted ten hours. Surgical resection is the mainstay of treatment because adjuvant therapy is unproven. The surgeon should make every effort to achieve complete resection of all gross disease, which may necessitate the removal of adjacent organs $(5 ; 6)$. The low incidence of lymph node metastases confirms that lymphadenectomy is not routinely warranted (2). Recurrence of GIST is usual, and one report found only $10 \%$ of patients to be free of disease after long-term follow-up (8). No evidence of disease recurrence after six months in this case.

\section{Conflict of interest: None}

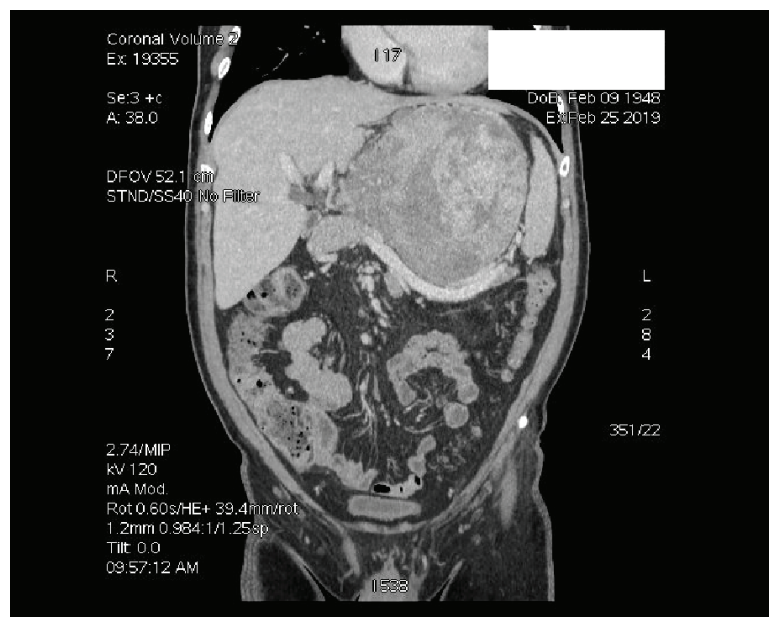

Fig. 1. Coronal CT image of the abdomen demonstrate a $21 \mathrm{~cm} \times 15 \mathrm{~cm} \times 15 \mathrm{~cm}$ tumor mass. Blood supply from truncus coeliacus

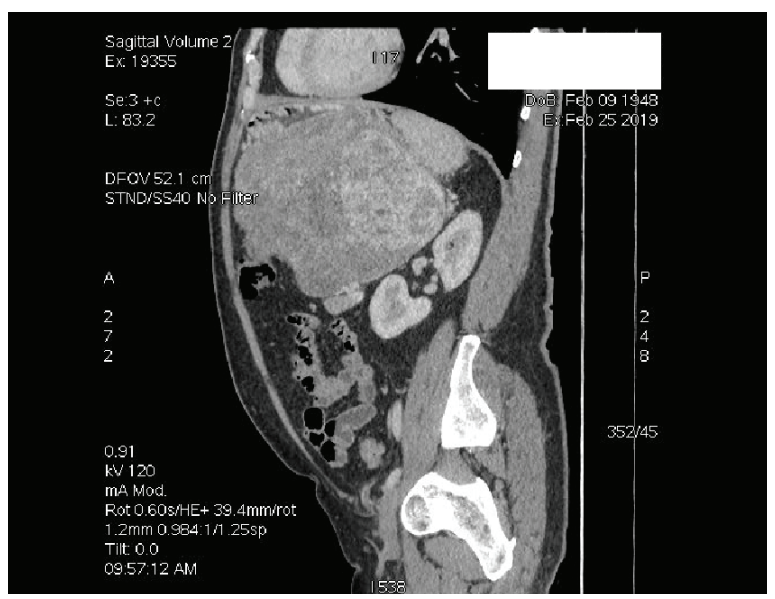

Fig. 2. Sagittal CT image, the stomach was compressed and dislocated by the tumor

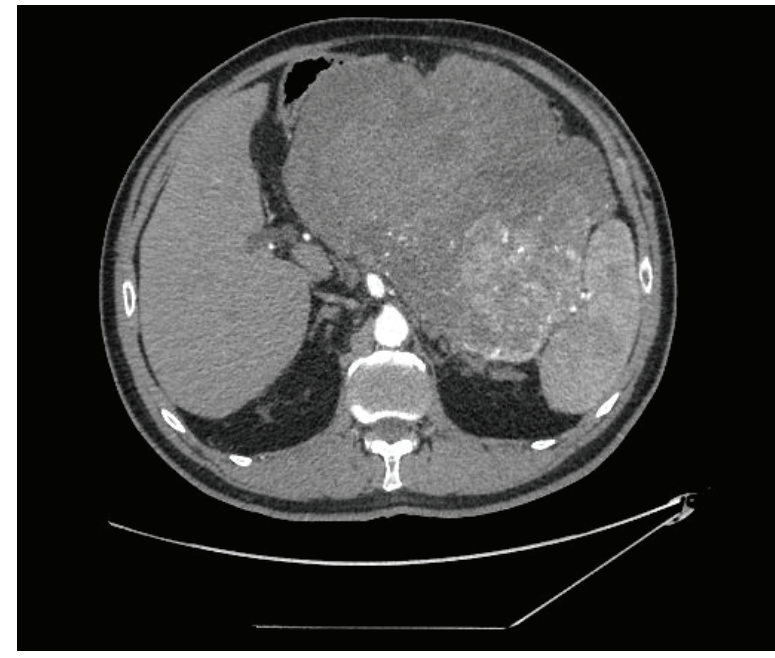

Fig. 3. Axial CT image, indistinct border between stomach back wall and the tumor

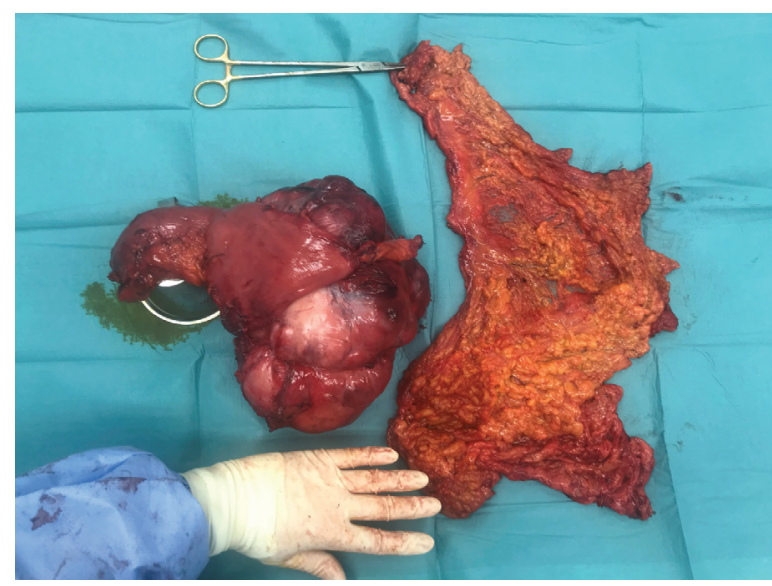

Fig. 4. Stomach with large tumor and epiploon 


\section{REFERENCES}

1. DeMatteo RP et all. Two hundred gastrointestinal stromal tumors: recurrence patterns and prognostic factors for survival. Ann Surg. 2000 Jan; 231(1):51-8.

2. Fong $\mathrm{Y}$ et all. Lymph node metastasis from soft tissue sarcoma in adults. Analysis of data from a prospective database of 1772 sarcoma patients. Ann Surg 1993; 217:72-77.

3. Fukuda $\mathrm{S}$ et all. Giant gastric gastrointestinal stromal tumor with severe peritoneal dissemination controlled by imatinib therapy following debulking surgery: a case report. J Med Case Rep. 2017 Feb 7; $11(1): 33$.

4. Fülöp E et all. Gastrointestinal stromal tumors: review on morphology, diagnosis and management. J Morphol Embryol. 2009; 50(3):319-26.

5. Harlan LC et all. Gastrointestinal stromal tumors: treatment patterns of a population-based sample. J Surg Oncol. 2015 May; $111(6): 702-7$.

6. Koyuncuer A et al. A rare case of giant gastrointestinal stromal tumor of the stomach involving the serosal surface. Int $\mathrm{J}$ Surg Case Rep 2015;12:90-4.
7. Novitsky YW et al. Long-term outcomes of laparoscopic resection of gastric gastrointestinal stromal tumors. Ann Surg. 2006 Jun; 243(6):73845; discussion 745-7.

8. Pollock RE et al. Prognostic implications of patterns of failure for gastrointestinal leiomyosarcomas. Cancer. 1992 Mar 15; 69(6):1334-41.

9. DemetriGD, et al. NCCN task force report: optimal management of patients with gastrointestinal stromal tumors (GIST) - expansion and update of NCCN clinical practice guidelines. J Natl Compr Cancer Netw. 2004;2:S1-S26.

\section{Address:}

Irina Liepina

Riga 1st Hospital,

Department of Surgery

Bruninieku street 5, LV-1001

Riga, Latvia

e-mail: irina.liepina@1slimnica.lv 